\title{
Prevalence, trend, determinanants and prediction of autism spectrum disorders among dubai population, diagnostic approach and management contexts
}

\begin{abstract}
Background: Autism spectrum disorder (ASD) is a public health concern in Dubai, Autism is the most common of the Pervasive Developmental Disorders in Dubai. Autism poses a particularly large public health challenge and an inspiring lifelong challenge for many families; it is a lifelong challenge of a different nature, it is a spectrum of neurodevelopment disorder, which often results in an array of motor impairments. These motor impairments often lead to reduced performance in activities of daily living as well as in societal tasks which require specific motor abilities and skills
\end{abstract}

Objectives: To study the prevalence's, determinants and prediction of Autism among Dubai population. To study some associated factors

Methodology: Retrospective records review approach has been applied, qualitative methodology (focus groups with mental health experts working at: Al Jalila hospital (AJH), Dubai Autism Centre (DAC), Dubai Rehabilitation Centre for Disabilities, Latifa hospital, Private Sector Healthcare (PSH).) Quantitative methodology through, Prevalence, incidence estimates based on international research (a systematic research review) along with expert interviews has been applied as well.

Results: The study showed that $84.9 \%$ of the cases were males and $51.9 \%$ of the autism cases were females, $30.9 \%$ were UAE nationals and $69.1 \%$ were expatriate, about $72.4 \%$ were diagnosed as Autism spectrum disorders, $9.2 \%$ as ASD features and $9.2 \%$ were diagnosed as other disorders when autism diagnostic test applied Dubai Autism Centre estimates it affects 1 in 146 births $(0.68 \%)$. By apply these estimates to the total number of births in Dubai for 2014, it is predicted there would be approximately 199 children (of which $58,(29.1 \%)$ were Nationals and $141,(70.9 \%)$ were Non-Nationals) suffering from autism at some stage. About $16.4 \%$ of children seek help for ASD assessment (through their families) between the age group 6-18+. It is critical to understand and address factors for seeking late-stage diagnosis, as ASD can be diagnosed much earlier and how many of these later presenters are actually diagnosed with ASD. Families do not consult GPs for early diagnosis for a variety of reasons including cultural reasons. The study reflected future prediction of the cases based on international standards prevalence and the population density as reflected by figure one which reflected the highest prevalence at the age intervals of 25-29 years, 30-34 years and 35-39 years respectively

Conclusion: Autism is still ongoing challenging public health issue in Dubai, in terms of raising up prevalence, incidences, trends, cases sorting out and management context of autism is one of actual national health care system gaps.

Recommendation: Effective school health strategies is needed and implemented by nurses who are qualified and experienced in identifying children with ASD. Training of GP's to aid early diagnosis of Autism and increase awareness. There is an urgent need for an adult autism centre for when the children leave the safe environment of the school at 18 years. There is a need for further studies to cover the needs of people with an Autism Spectrum Disorder (ASD)

Keywords: autism spectrum, dubai population, prevalence, prediction
Volume 7 Issue I - 2017

\author{
Kadim Al abbady,' Hamid Yahya Hessian, ${ }^{2}$ \\ Mohamed Wasif Alaam ${ }^{3}$ \\ 'Public Health and safety Dept, Dubai Health Authority, UAE \\ 2Primary Health Care, Dubai Health authority, UAE
}

Correspondence: Hamid yahya Hessian, Primary Health Care, Dubai Health authority, UAE, Email hyHussain@dha.gov.ae

Received: July 27, 2017 | Published: August 10, 2017

\section{Background}

Autism poses a particularly large public health challenge and an inspiring lifelong challenge for many families; it is a lifelong challenge of a different nature. It is a spectrum of neurodevelopment disorder, which often results in an array of motor impairments. These motor impairments often lead to reduced performance in activities of daily living as well as in societal tasks which require specific motor abilities and skills. Global prevalence estimates of autism spectrum disorders found a median of 62 cases per 10,000 people. ${ }^{1}$ There is a lack of evidence from low- and middle-income countries though. ${ }^{1}$ ASD averages a 4.3:1 male-to-female ratio. ${ }^{2}$ The number of children known to have autism has increased dramatically since the 1980 s, at least partly due to changes in diagnostic practice; it is unclear whether prevalence has actually increased; ${ }^{2}$ and as-yet-unidentified environmental risk factors cannot be ruled out. ${ }^{3}$ The risk of autism is associated with several prenatal factors, including advanced paternal age and diabetes in the mother during pregnancy. ${ }^{4} \mathrm{ASD}$ is associated with several genetic disorders ${ }^{5}$ and with epilepsy. ${ }^{6}$ 
On 2006 study of nearly 57,000 British nine- and ten-year-old reported a prevalence of 3.89 per 1,000 for autism and 11.61 per 1,000 for ASD; these higher figures could be associated with broadening diagnostic criteria. ${ }^{7}$ Studies based on more-detailed information, such as direct observation rather than examination of medical records, identify higher prevalence; this suggests that published figures may underestimate ASD's true prevalence. ${ }^{8}$ A 2009 study of the children in Cambridgeshire, England used different methods to measure prevalence, and estimated that $40 \%$ of ASD cases go undiagnosed, with the two least-biased estimates of true prevalence being 11.3 and 15.7 per $1,000 .{ }^{9}$ On 2009 U.S. study based on 2006 data estimated the prevalence of ASD in 8-year-old children to be 9.0 per 1,000 (approximate range 8.6-9.3). ${ }^{2}$. A 2009 report based on the 2007 Adult Psychiatric Morbidity Survey by the National Health Service determined that the prevalence of ASD in adults was approximately $1 \%$ of the population, with a higher prevalence in males and no significant variation between age groups..$^{10}$ These results suggest that prevalence of ASD among adults is similar to that in children and rates of autism are not increasing. ${ }^{11-13}$

\section{Objectives}

To study the prevalence's, determinants and prediction of Autism among Dubai population. To study some associated factors.

\section{Methodology}

Retrospective records review approach has been applied, qualitative methodology (focus groups with mental health experts working at: Al Jalila hospital (AJH), Dubai Autism Centre (DAC), Dubai Rehabilitation Centre for Disabilities, Latifa hospital, Private Sector Healthcare (PSH).) Quantitative methodology through, Prevalence, incidence estimates based on international research (a systematic research review) along with expert interviews has been applied as well.

\section{Results}

The study revealed that prevalence of Autism is steadily rising along the last 4 years as per cases registry at Dubai autism centre for both national and expatriate population, the study showed that about two third of autism cases in Dubai is among expatriate population and on third among UAE national population as reflected by table. The study reflected future prediction of the cases based on international standards prevalence and the population density as reflected by figure one which reflected the highest prevalence at the age intervals of 25-29 years, 30-34 years and 35-39 years respectively as shown by figure. ${ }^{1}$

Table I Prevalence of Autism cases among Dubai Population by nationality and years

\begin{tabular}{llll}
\hline & Nationals & Non-Nationals & Total \\
\hline 2011 & 60 & 119 & 179 \\
2012 & 61 & 130 & 191 \\
2013 & 60 & 138 & 198 \\
2014 & 58 & 141 & 199 \\
\hline
\end{tabular}

The study showed the $84.9 \%$ of the cases were males and $51.9 \%$ of the autism cases were females, $30.9 \%$ were UAE nationals and $69.1 \%$ were expatriate, about $72.4 \%$ were diagnosed as Autism spectrum disorders, $9.2 \%$ as ASD features and $9.2 \%$ were diagnosed as other disorders when autism diagnostic test applied for assessment as shown by table. ${ }^{2}$
Table 2 Dubai Autism Centre (DAC) assessment statistics during September 20I4-June 2015

\begin{tabular}{llll}
\hline & & Number & $\%$ \\
\hline \multirow{2}{*}{ Gender } & Male & 129 & 84.9 \\
& Female & 23 & 15.1 \\
& U.A.E. & 47 & 30.9 \\
Residence & Non-U.A.E. & 105 & 69.1 \\
& U.A.E. & 128 & 84.2 \\
& Non-U.A.E. & 24 & 15.8 \\
Diagnosis & ASD & 110 & 72.4 \\
& ASD features & 14 & 9.2 \\
& Other & 28 & 18.4 \\
Type of & Full -2 parts & 62 & 40.8 \\
assessment & assessment & & \\
& Only short form for & 90 & 59.2 \\
Short form of & screening tool & & \\
assessment for & ASD & 48 & 31.6 \\
screening tool & Other & 16 & 10.5 \\
Total & & 26 & 17.1 \\
& & 152 & $100 \%$ \\
\hline
\end{tabular}

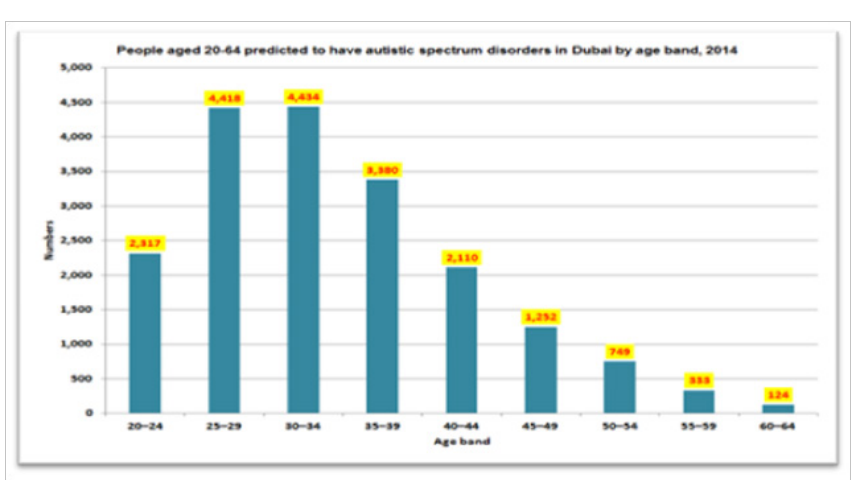

Figure I Predicted Autism cases among Dubai population according to age.

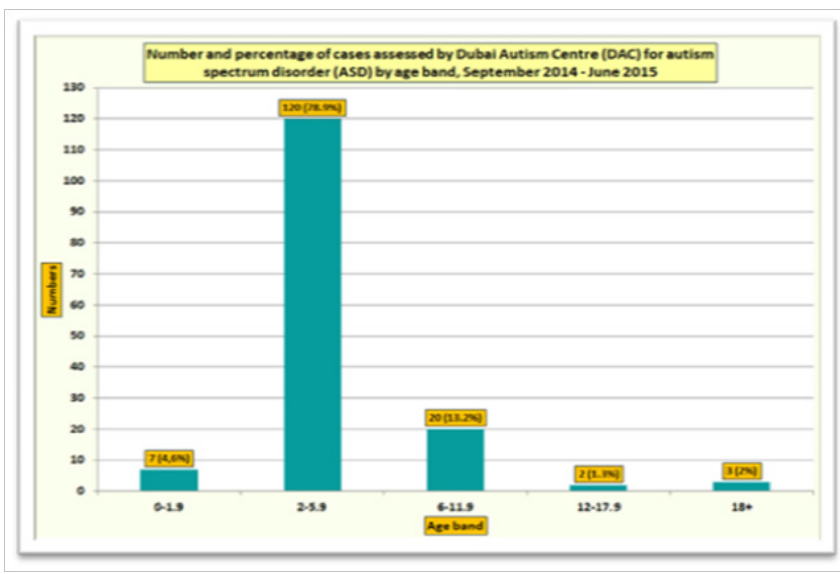

Figure 2 Autism cases assessed by Dubai Autism centre in Dubai.

The current study adopt a novel approach to estimating the prevalence of these conditions and presents strong evidence that undiagnosed cases do indeed exist in the school-aged population Similar to other studies, The estimates of known cases reported in this paper accord with previous prevalence estimates ${ }^{14-16}$ converging at around $1 \%$. Further, prevalence estimates reported from other countries fall within the confidence intervals of our estimates reported in this paper. ${ }^{17-19}$ 


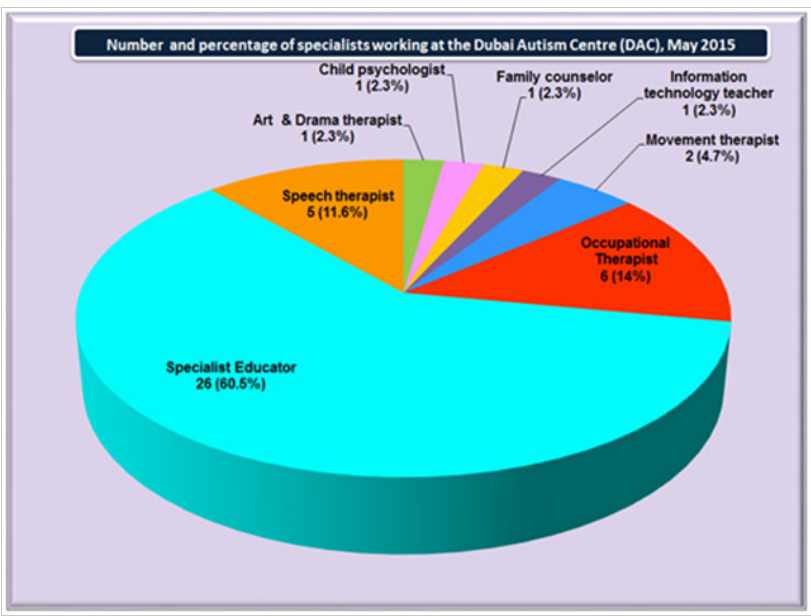

Figure 3 Management approach ay Dubai Autism centre by roles and function.

Similar the current study which showed increasing trend of prevalence along the last four years, many of other worldwide publication showed that, the prevalence of autism is increasing with time. Earlier prevalence estimates were lower, centering at about 0.5 per 1,000 for autism during the 1960s and 1970s and about 1 per 1,000 in the 1980s, as opposed to today's 1-2 per 1000.21].

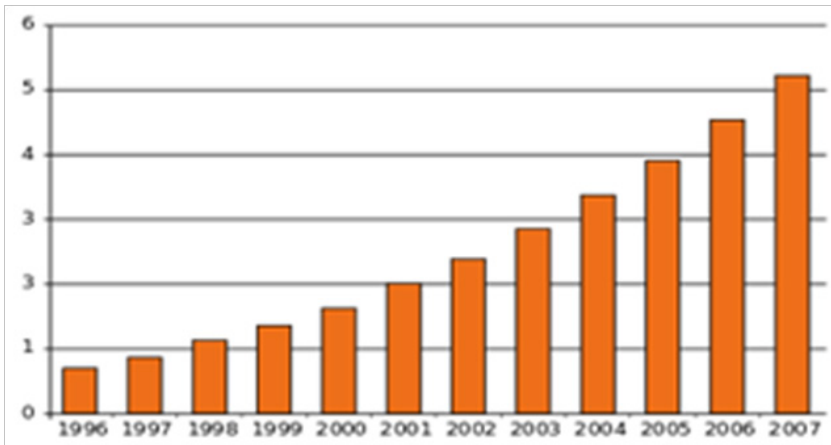

Reports of autism cases per 1,000 children grew dramatically in the U.S. from 1996 to 2007. It is unknown how much, if any, growth came from changes in autism's prevalence. ${ }^{20}$ The number of reported cases of autism increased dramatically in the 1990s and early 2000 s, prompting investigations into several potential reasons. ${ }^{21}$ More children may have autism; that is, the true frequency of autism may have increased. There may be more complete pickup of autism (case finding), as a result of increased awareness and funding. For example, attempts to sue vaccine companies may have increased casereporting. The diagnosis may be applied more broadly than before, as a result of the changing definition of the disorder, particularly changes in DSM-III-R and DSM-IV. An editorial error in the description of the PDD-NOS category of Autism Spectrum Disorders in the DSMIV, in 1994, inappropriately broadened the PDD-NOS construct. The error was corrected in the DSM-IV-TR, in 2000, reversing the PDD-NOS construct back to the more restrictive diagnostic criteria requirements from the DSM-III-R..$^{22}$ Successively earlier diagnosis in each succeeding cohort of children, including recognition in nursery (preschool), may have affected apparent prevalence but not incidence. A review of the "rising autism" figures compared to other disabilities in schools shows a corresponding drop in findings of mental retardation. ${ }^{23}$ The reported increase is largely attributable to changes in diagnostic practices, referral patterns, availability of services, age at diagnosis, and public awareness. ${ }^{24-27}$ A widely cited 2002 pilot study concluded that the observed increase in autism in California cannot be explained by changes in diagnostic criteria, ${ }^{28}$ but a 2006 analysis found that special education data poorly measured prevalence because so many cases were diagnosed, and that the 1994-2003 U.S. increase was associated with declines in other diagnostic categories, indicating that diagnostic substitution had occurred. ${ }^{29}$ A 2007 study that modeled autism incidence found that broadened diagnostic criteria, diagnosis at a younger age, and improved efficiency of case ascertainment, can produce an increase in the frequency of autism ranging up to 29-fold depending on the frequency measure, suggesting that methodological factors may explain the observed increases in autism over time..$^{30} \mathrm{~A}$ small 2008 study found that a significant number $(40 \%)$ of people diagnosed with pragmatic language impairment as children in previous decades would now be given a diagnosis as autism. ${ }^{31}$ A study of all Danish children born in 1994-99 found that children born later were more likely to be diagnosed at a younger age, supporting the argument that apparent increases in autism prevalence were at least partly due to decreases in the age of diagnosis. A 2009 study of California data found that the reported incidence of autism rose 7- to 8-fold from the early 1990s to 2007, and that changes in diagnostic criteria, inclusion of milder cases, and earlier age of diagnosis probably explain only a 4.25 -fold increase; the study did not quantify the effects of wider awareness of autism, increased funding, and expanding treatment options resulting in parents' greater motivation to seek services. ${ }^{29}$ Another 2009 California study found that the reported increases are unlikely to be explained by changes in how qualifying condition codes for autism were recorded. ${ }^{31}$ Several environmental risk factors have been proposed to support the hypothesis that the actual frequency of autism has increased. These include certain foods, infectious disease, and pesticides. There is overwhelming scientific evidence against the MMR hypothesis and no convincing evidence for the thiomersal (or Thimerosal) hypothesis, so these types of risk factors have to be ruled out.

\section{Conclusion}

Autism is still ongoing challenging public health issue in Dubai, in terms of raising up prevalence, incidences, trends, cases sorting out and management context of autism is one of actual national health care system gaps.

\section{Recommendation}

Effective school health strategies is needed and implemented by nurses who are qualified and experienced in identifying children with ASD. Training of GP's to aid early diagnosis of Autism and increase awareness. There is an urgent need for an adult autism centre for when the children leave the safe environment of the school at 18 years, there is a need for further studies to cover the needs of people with an Autism Spectrum Disorder (ASD).

\section{Ackowledgments}

None.

\section{Conflicts of interest}

The author declares no conflicts of interest.

\section{References}

1. Elsabbagh M, Divan G, Koh YJ, et al. Global prevalence of autism and other pervasive developmental disorders. Autism Res. 2012;5(3):160 179. 
2. Newschaffer CJ, Croen LA, Daniels J, et al. The epidemiology of autism spectrum disorders. Annu Rev Public Health. 2007;28:235-258.

3. Rutter M. Incidence of autism spectrum disorders:changes over time and their meaning. Acta Pediatric. 2005;94(1):2-15.

4. Gardener H, Spiegel man D, Buka SL. Prenatal risk factors for autism:comprehensive meta-analysis. Br J Psychiatry. 2009;195(1):714

5. Zafeiriou DI, Ververi A, Vargiami E. Childhood autism and associated co morbidities. Brain Dev. 2007;29(5):257-272.

6. Levisohn PM. The autism-epilepsy connection. Epilepsia. 2007;48(Supp 9):33-35.

7. Prevalence of autism spectrum disorders - autism and developmental disabilities monitoring network, 14 sites, United States, 2008. MMWR Surveill Summ. 61(3):1-19.

8. Blumberg SJ, Bramlett MD, Kogan MD, et al. Changes in prevalence of parent-reported autism spectrum disorder in school-aged U.S. children: 2007 to 2011-2012 (PDF). Natl Health Stat Report. 2013;65:1-11.

9. Brugha T, Cooper SA, McManus S, et al. Estimating the prevalence of autism spectrum conditions in adults:extending the 2007 Adult Psychiatric Morbidity Survey" (PDF). The Information Centre for Health and Social Care. National Health Service, UK. 2012.

10. Geschwind DH. Autism:many genes, common pathways?. Cell. 2008;135(3):391-395

11. ICD-10: International Statistical Classification of Diseases and Related Health Problems. Tenth Revision. World Health Organization. 2007.

12. Pinel JPG. Biopsychology. Boston, Massachusetts: Pearson. 2011. p. 235.

13. Rogers SJ. What are infant siblings teaching us about autism in infancy? Autism Res. 2009;2(3):125-137.

14. Baird G, Simonoff E, Pickles A, et al. Prevalence of disorders of the autism spectrum in a population cohort of children in South Thames:the Special Needs and Autism Project (SNAP). Lancet. 2006;368(9531):210215.

15. Bertrand J, Mars A, Boyle C, et al. Prevalence of autism in a United States population:the Brick Township, New Jersey, investigation. Pediatrics. 2001;108(5):1155-1161.

16. Rutter M. Aetiology of autism:findings and questions. J Intellect Disabil Res. 2005;49(4):231-238

17. Fombonne E. The changing epidemiology of autism. J Applied Res Intell Dis. 2005;18(4):281-294.
18. Gillberg C, Cederlund M, Lamberg K, et al. Brief report:' the autism epidemic'. The registered prevalence of autism in a Swedish urban area. J Autism Dev Disord. 2006;36(3):429-435

19. Kadesjo B, Gillberg C, Hagberg B. Brief report: autism and Asperger syndrome in seven-year-old children:a total population study. J Autism Dev Disord. 1999;29(4):327-331.

20. Fombonne E. Prevalence and changes in diagnostic practice:The prevalence of autism. JAMA. 2003;289(1):87-89.

21. Wing L, Potter D. The epidemiology of autistic spectrum disorders:is the prevalence rising? Ment Retard Dev Disabil Res Rev. 2002;8(3):151161

22. Wing L, Potter D. National Autistic Society. Notes on the prevalence of autism spectrum disorders. 1999.

23. Clarification of the definition of Pervasive Developmental Disorder Not Otherwise Specified". Five Easy Graphs, USDE figures.

24. Byrd RS, Sage AC, Keyzer J, et al. "Report to the legislature on the principal findings of the epidemiology of autism in California:a comprehensive pilot study". M.I.N.D. Institute, USA, 2002. p. 70.

25. Shattuck PT. The contribution of diagnostic substitution to the growing administrative prevalence of autism in US special education. Pediatrics. 2006;117(4):1028-1037.

26. Wazana A, Bresnahan M, Kline J. The autism epidemic:fact or artifact? J Am Acad Child Adolesc Psychiatry. 2007;46(6):721-730.

27. Bishop DVM, Whitehouse AJO, Watt HJ, et al. Autism and diagnostic substitution:evidence from a study of adults with a history of developmental language disorder. Dev Med Child Neurol. 2008;50(5):341-345.

28. Parner ET, Schendel DE, Thorsen P. Autism prevalence trends over time in Denmark: changes in prevalence and age at diagnosis. Arch Pediatr Adolesc Med. 2008;162(12):1150-1156.

29. Hertz Picciotto I, Delwiche L. The rise in autism and the role of age at diagnosis. Epidemiology. 2009;20(1):84-90.

30. Grether JK, Rosen NJ, Smith KS, et al. Investigation of shifts in autism reporting in the California Department of Developmental Services. $J$ Autism Dev Disord. 2009;39(10):1412-1419.

31. Szpir M. Tracing the origins of autism:a spectrum of new studies Environ Health Perspect. 2006;114(7):A412-A418. 